\title{
Automatic and reliable quantification of tonic dopamine concentrations in vivo using a novel probabilistic inference method
}

\author{
Jaekyung Kim ${ }^{1,2}$, Abhijeet S. Barath ${ }^{3}$, Aaron E. Rusheen ${ }^{3,4}$, \\ Juan M. Rojas Cabrera ${ }^{3}$, J. Blair Price ${ }^{3}$, Hojin Shin ${ }^{3}$, Abhinav Goyal ${ }^{3,5}$, Jason W. Yuen ${ }^{3}$, \\ Danielle E. Jondal ${ }^{3}$, Charles D. Blaha ${ }^{3}$, Kendall H. Lee ${ }^{3,6}$, Dong Pyo Jang ${ }^{7}$, Yoonbae $\mathrm{Oh}^{3,6^{*}}$
}

${ }^{1}$ Department of Neurology, University of California-San Francisco, San Francisco, CA, 94158, USA

${ }^{2}$ Neurology and Rehabilitation Service, San Francisco Veterans Affairs Medical Center, San Francisco, CA, 94158, USA

${ }^{3}$ Department of Neurologic Surgery, Mayo Clinic, Rochester, MN 55905, USA

${ }^{4}$ Mayo Clinic Alix School of Medicine, Mayo Clinic, Rochester, MN 55905, USA

${ }^{5}$ Mayo Clinic Graduate School of Biomedical Sciences, Mayo Clinic, Rochester, MN 55905

${ }^{6}$ Department of Biomedical Engineering, Mayo Clinic, Rochester, MN 55905, USA

${ }^{7}$ Department of Biomedical Engineering, Hanyang University, Seoul, Republic of Korea

*Correspondence:

Dr. Yoonbae $\mathrm{Oh}, \mathrm{PhD}$

Mailing address: 200 First St, SW, Rochester, MN, 55905

Phone: 507-293-7992

Email: Oh.Yoonbae@Mayo.edu 
Electrode fabrication.

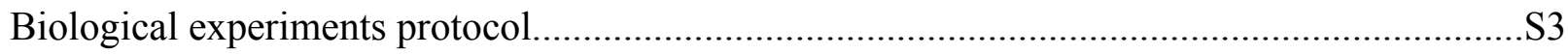

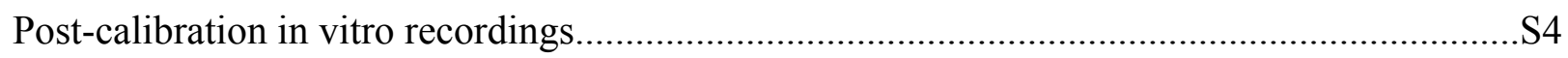

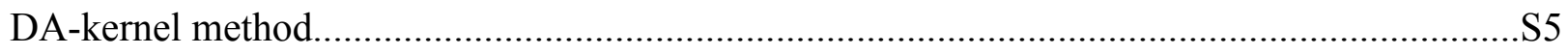

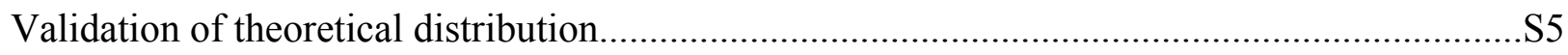

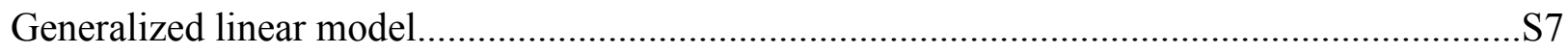

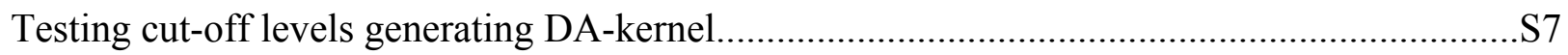




\section{Electrode fabrication}

The carbon-fiber microelectrode design was modified from our previous studies ${ }^{1-2}$. Single carbon fibers

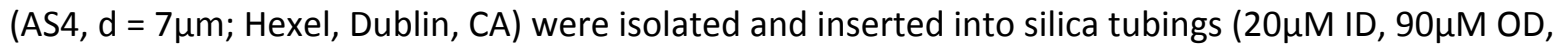
$10 \mu \mathrm{M}$ coat with polyimide; Polymicro Technologies, Phoenix, AZ) to manufacture the carbon-fiber microelectrodes. The connection between the carbon fiber and the silica tubings was sealed with polyamic acid (Sigma-Aldrich, St. Louis, MO). They were heated to $200^{\circ} \mathrm{C}$ to polymerize the polyamic acid into a polyimide film. A silver-based conductive paste was then used to attach the silica tubing to a nitinol (Nitinol \#1, an alloy of nickel and titanium; Fort Wayne Metals, IN) extension wire ${ }^{1}$. A polyimide tubing (0.0089"ID, 0.0134"OD, 0.00225" WT; Vention Medical, Salem, NH) was then used to insulate the nitinol wire and its attached carbon fiber except at the carbon fiber sensing part. We trimmed the exposed carbon fiber under a dissecting microscope to a length of approximately $50 \mu \mathrm{m} . \mathrm{An} \mathrm{Ag} / \mathrm{AgCl}$ reference electrode was prepared by chlorinating the exposed tip of a Teflon-coated silver wire (A-M systems, Inc., Sequim, WA) in saline with a 9 V dry cell battery.

\section{Biological experiments protocol}

Rats were housed in a AAALAC accredited vivarium $\left(21^{\circ} \mathrm{C}, 45 \%\right.$ humidity $)$ with a $12 \mathrm{hr}$ light-dark cycle (lights on at $0600 \mathrm{hr}$ ) with ad libitum access to food and water. They were anesthetized with urethane $(1.6 \mathrm{~g} / \mathrm{kg}$, i.p.) and stabilized in a commercially available stereotaxic frame (David Kopf Instruments, Tujunga, CA) for the surgery. A longitudinal skin incision was made on the top of the head to expose the skull and three burr holes $(0.5-1.0 \mathrm{~mm}$ diameter $)$ were made for the implantation of a carbon-fiber microelectrode, a bipolar electrical stimulating electrode (Plastic One, MS303/2, Roanoke, VA, USA) and an $\mathrm{Ag} / \mathrm{AgCl}$ reference electrode. The reference electrode was placed superficially in cortex contralateral to the carbon-fiber microelectrode and stimulating

electrode site. Electrode coordinates were referenced by Watson and Paxinos rat brain atlas ${ }^{3}$ based on flat-skull position using bregma and dura as reference points with coordinates anteroposterior 
(AP), mediolateral (ML), and dorsoventral (DV). The carbon-fiber microelectrode was placed in the dorsomedial striatum (AP $+1.0 \mathrm{~mm}$; $\mathrm{ML}+2.5 \mathrm{~mm}$; DV -4.5 to $-5.5 \mathrm{~mm}$ ) of the right hemisphere. The stimulating electrode was inserted ipsilaterally just above the medial forebrain bundle (MFB, AP -4.8; ML +1.0; DV -8.0 to -9.0). A train of bipolar pulses (2 ms pulse width, $200 \mu \mathrm{A}, 60 \mathrm{~Hz}$ ) using WINCS Harmoni electrometer was delivered for 2 seconds to identify the optimal dopamine (DA) release sites in the striatum. FSCV signal was synchronized with electrical stimulation by interleaving the intervals of stimulation during FSCV scans to prevent stimulation artefact ${ }^{4}$. Thus, electrical stimulation was not applied when the FSCV pulses (about 10ms) were delivered. The carbon-fiber microelectrode and the electrical stimulating electrode were gradually adjusted until a robust phasic DA signal was detected at the carbon-fiber microelectrode using FSCV. Immediately thereafter, we switched to MCSWV recording. The MCSWV waveform was applied at $0.1 \mathrm{~Hz}$ for the duration of recording. Stabilization of the recorded electrochemical signal was achieved in the first 10 minutes.

\section{Post-calibration in vitro recordings}

DA $\mathrm{HCl}$ was dissolved in distilled water at a stock concentration of $1 \mathrm{mM}$ and preserved in $0.1 \mathrm{M}$ perchloric acid. Samples from the stock solutions were diluted to the desired concentration with TRIS buffer (15mM tris, $3.25 \mathrm{mM} \mathrm{KCl,} 140 \mathrm{mM} \mathrm{NaCl}, 1.2 \mathrm{mM} \mathrm{CaCl} 2,1.25 \mathrm{mM} \mathrm{NaH} 2 \mathrm{PO} 4$, 1.2mM MgCl2, and 2.0mM Na2SO4, with the $\mathrm{pH}$ adjusted to 7.4). Immediately after in vivo experiments, post-calibration was performed in vitro. The carbon-fiber microelectrode and reference electrode used for the in vivo experiment were placed in a beaker with TRIS buffer. MCSWV was applied for 10 minutes to stabilize the signal and DA (200nM, 500nM and $1000 \mathrm{nM}$ ) was added to the beaker. A disposable pipette was used to mix the solutions. The recording was continued for approximately 15 minutes after addition of DA. All chemicals were purchased from Sigma-Aldrich (St. Louis, MO). 


\section{DA-kernel method}

A threshold algorithm was applied to the post-calibration in vitro and in vivo DA response of MCSWV, where signals greater than cut-off level $(60 \%$ of the peak current for the peak-based method; top $5^{\text {th }}$ percentile of the oxidation currents distribution for the probabilistic inference method) in the $2 \mathrm{D}$ voltammogram were given a logical value of 1 , while others were given a logical value of 0 (yellow and red, respectively, in Figure 2B). The resulting mask was called the dopamine-kernel, i.e., DA-kernel. A DA-kernel was computed for a respective in vivo and postcalibration in vitro recording of each animal. We computed the DA-kernel and applied it to the $2 \mathrm{D}$ voltammogram by element-wise multiplication. This multiplication computation results in selecting the currents over the entire area of value 1 in DA-kernel; in other words, extracting closely DA-related signals. The results were then integrated and used to predict the DA concentration. This process was repeated for each $2 \mathrm{D}$ voltammogram to obtain the trend of DA over time (Figure 2C).

\section{Validation of analytical distribution}

The lognormal distribution was used to estimate an analytical distribution of the post-calibration in vitro and in vivo DA response of MCSWV (Figure 3). To assess if the empirical MCSWV data plausibly from the lognormal distribution, we performed a graphical method, a quantile-quantile (Q-Q) plot (Figure S1 A and B). We could confirm that Q-Q points fall along a unity line in Q-Q plot for both in vitro and in vivo data of all six animals, which provides strong evidence that the empirical data fairly come from a lognormal distribution. We also compared the fitting of the empirical data with lognormal distribution to the fitting with a gamma distribution (Figure S1C). The mean of RMSE (root mean squared error) of the theoretical distribution was lower using the lognormal function compared to using the gamma function, which shows the lognormal distribution greater estimator of the theoretical distribution of the DA response of MCSWV. Furthermore, we performed a likelihood ratio test of fitting 
using the lognormal distribution against using the gamma distribution. This test supported that lognormal distribution is greater theoretical estimator (six electrodes in vitro post-calibrations: likelihood ratio statistics $>3.4 \times 10^{2}, \mathrm{P}<1.1 \times 10^{-10}$; six rats in vivo: likelihood ratio statistics $>45, \mathrm{P}<2.3 \times 10^{-9}$ ).

A

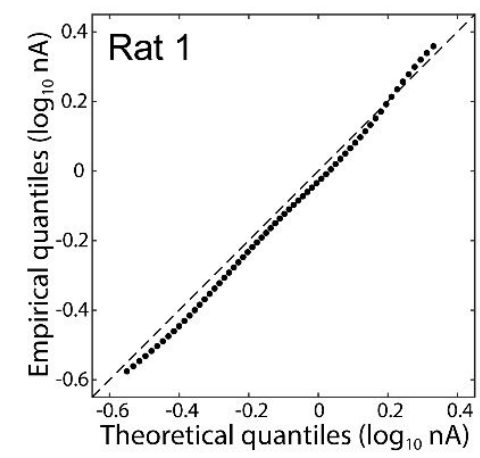

B

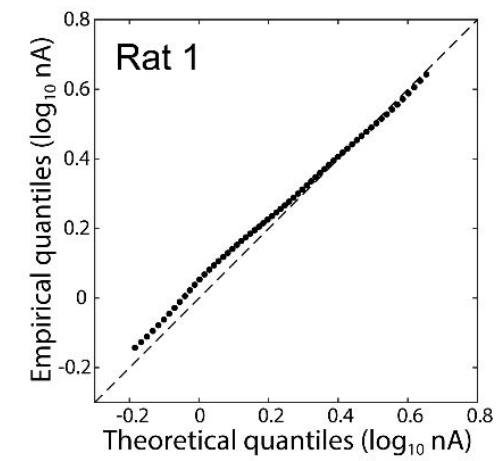

C
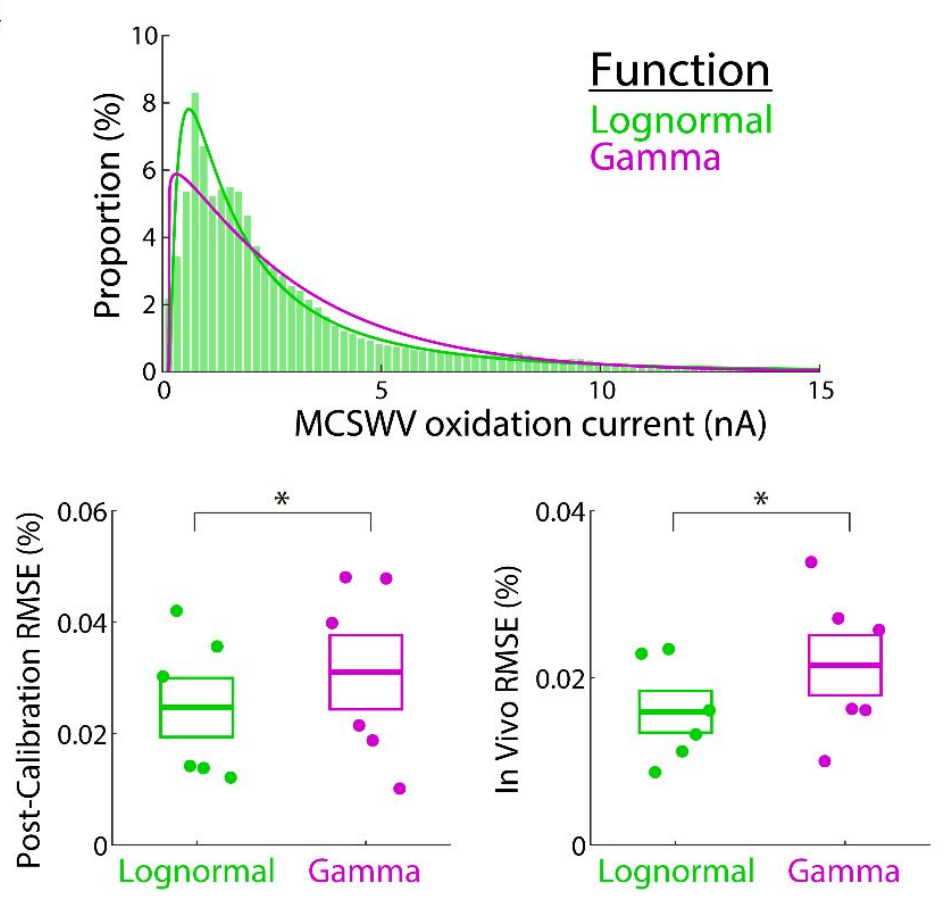

Figure S1. Validation of fitting empirical data with lognormal distribution.

(A and B) quantile-quantile (Q-Q) plots to assess if a set of empirical data came from the theoretical distribution (i.e. lognormal distribution). The representative examples of in vitro post-calibration (A) and in vivo (B) data of two rats are shown. The data of "Rat 1 " correspond to the data shown in Figure 2-4.

(C) Comparison of fitting the Rat 1 data with lognormal function and gamma function. Lognormal functions were significantly better to estimate theoretical distribution of the MCSWV oxidation current for both in vitro postcalibration (RMSE: lognormal function: $0.025 \pm 0.005 \%$; gamma function: $0.031 \pm 0.007 \%$; lognormal vs. lognormal, paired t-test, $\mathrm{t}_{5}=-3.21$, $\mathrm{P}=0.024)$ and in vivo (RMSE:

lognormal function: $0.016 \pm 0.003 \%$; gamma function: $0.022 \pm 0.004 \%$; lognormal vs. lognormal, paired t-test, $\left.\mathrm{t}_{5}=-3.54, \mathrm{P}=0.016\right)$. Mean in vertical bar \pm SEM in box. 


\section{Generalized linear model}

To estimate the DA concentrations, we fit regression models using the quadratic function (secondorder polynomial function) as

$$
f(x)=a x^{2}+b x+c
$$

where $\mathrm{a}, \mathrm{b}$, and $\mathrm{c}$ are real numbers and $\mathrm{a} \neq 0$. This fitting regression model was implemented using the generalized linear mode (GLM). We used "fitglm" function in Matlab with the quadratic model. An individual GLM derived for each animal (thus, total six GLMs were generated in this study) was then used to predict DA concentrations The GLM was generated from in vitro post-calibration dataset. To compare the DA estimation methods (peak-based method vs probabilistic inference method), the full in vitro dataset was split into a training dataset (50\% of the total in vitro MCSWV scans) and a test dataset (50\% of the total in vitro MCSWV scans). Then, the GLM generated by the training dataset was used to predict DA concentrations for the test dataset including the $50 \%$ in vitro MCSWV scans as well as full in vivo MCSWV scans. To fit each GLM (total six models for six rats), we performed 5-fold cross-validation on the training set. We selected best-fitting parameters that returned the lowest cross-validated prediction error (measured as root mean squared error).

For the quadratic regression, in detail, we used in vitro MCSWV scans collected as the postcalibration procedure. The previous study demonstrated that the MCSWV responses to 50-1000 $\mathrm{nM}$ of DA were fitted well with quadratic function (Figure 1C) $)^{5}$. Thus, we fitted the regression model (i.e. link function of GLM) using the quadratic function ("quadratic" in the options of Matlab fitglm function). The integration of the MCSWV oxidation currents which overlapped with the predetermined binary DA-kernel was then computed. In individual animals, the integrated oxidation currents were normalized according to the area of the DA-kernel. The resultant link function (i.e., a fitted quadratic model with equation 1) was used to predict DA concentrations of the test dataset. More precisely, the GLM-based quadratic regression allows the model (i.e., link function) to be related to the response/dependent variable (i.e., DA concentration), by allowing the magnitude of the variance for each DA measurement to be a function of the predictor/independent variable (i.e., the integration of the oxidation currents within the DA-kernel).

\section{Testing cut-off levels generating DA-kernel}

We repeated the estimation of in vivo tonic DA concentration shown in Figure 5B using three different cut-off levels, top 1-2, 5, and $10 \%$ (Figure S2). For two animals, the top $1 \%$ of analytical distribution was out of the range of empirical data. In those cases, the top $2 \%$ was used for the cut-off level to generate DA-kernel. The probabilistic inference method using the cut-off levels of top $1-10 \%$ showed significantly reduced variance in the tonic DA level prediction across animals compared to the peak-based method. The cut-off at top 1-2\% appears to lose too much DArelated information in MCSWV responses and resulted in sparse predictions in tonic DA. Moreover, we could not find strong evidence that the cut-off of the top $5 \%$ is better than the cut-off of the top $10 \%$. However, in the within-animal comparison, more animals demonstrated significantly reduced variance 
using the cut-off of the top 5\% versus the top 10\% (significantly reduced variance in 5 rats using the top 5\% vs. 3 rats using the top 10\%). The selection of DA-related information is crucial for DA level estimations not only in FSCV and MCSWV techniques ${ }^{5-6}$. The cut-off level of top 5th percentile may provide a statistically DA-related signal from the analytically estimated distribution and less arbitrary standard to determine the DA-kernel based on statistical model (Figure 3 and 4B). This is analogous to a one-sided statistical test with a $5 \%$ significance level $^{7}$.

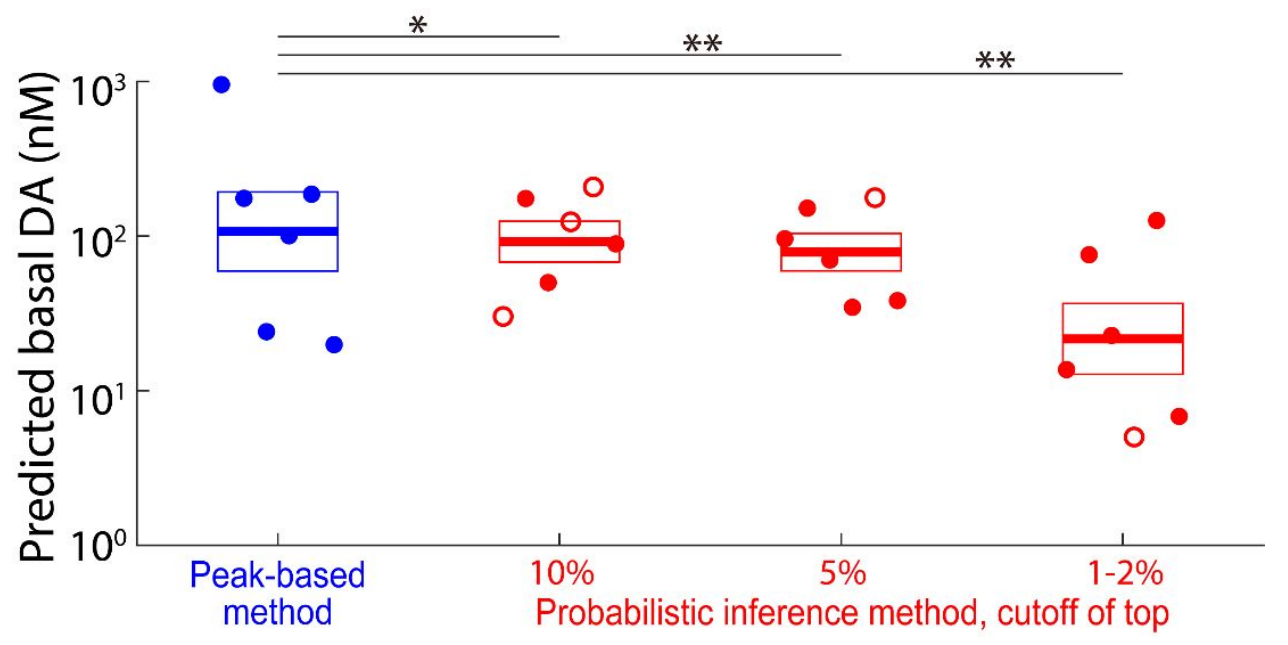

Figure S2. The predicted in vivo tonic DA concentrations with three different cut-off levels.

Variance of the predicted mean tonic DA across rats was significantly lower in the probabilistic inference method compared to the peak-based method (Bartlett's test, ${ }^{*} \mathrm{P}<0.05,{ }^{*} \mathrm{P}<0.01$; mean in vertical bar \pm SEM in box at $\log _{10}$ scale). Filled circles indicate significantly lower variance of tonic DA predictions within single animal using the probabilistic inference method compared to the peak-based method (Bartlett's test, $\mathrm{P}<0.05$; open circles indicate non-significance in Bartlett's test). 
1. Chang, S.-Y.; Kimble, C. J.; Kim, I.; Paek, S. B.; Kressin, K. R.; Boesche, J. B.; Whitlock, S. V.; Eaker, D. R.; Kasasbeh, A.; Horne, A. E., Development of the Mayo Investigational Neuromodulation Control System: toward a closed-loop electrochemical feedback system for deep brain stimulation. J neurosurg 2013, 119 (6), 1556-1565.

2. $\quad$ Chang, S.-Y.; Kim, I.; Marsh, M. P.; Jang, D. P.; Hwang, S.-C.; Van Gompel, J. J.; Goerss, S. J.; Kimble, C. J.; Bennet, K. E.; Garris, P. A. In Wireless fast-scan cyclic voltammetry to monitor adenosine in patients with essential tremor during deep brain stimulation, Mayo Clin Proc, Elsevier: 2012; pp 760-765. 3. Paxinos, G.; Watson, C., A stereotaxic atlas of the rat brain. New York: Academic 1998.

4. Lee, K. H.; Lujan, J. L.; Trevathan, J. K.; Ross, E. K.; Bartoletta, J. J.; Park, H. O.; Paek, S. B.; Nicolai, E. N.; Lee, J. H.; Min, H.-K., WINCS Harmoni: Closed-loop dynamic neurochemical control of therapeutic interventions. Sci rep 2017, 7, 46675.

5. Oh, Y.; Heien, M. L.; Park, C.; Kang, Y. M.; Kim, J.; Boschen, S. L.; Shin, H.; Cho, H. U.; Blaha, C. D.; Bennet, K. E.; Lee, H. K.; Jung, S. J.; Kim, I. Y.; Lee, K. H.; Jang, D. P., Tracking tonic dopamine levels in vivo using multiple cyclic square wave voltammetry. Biosen Bioelectron 2018, 121, 174-182.

6. Atcherley, C. W.; Wood, K. M.; Parent, K. L.; Hashemi, P.; Heien, M. L., The coaction of tonic and phasic dopamine dynamics. Chem Commun 2015, 51 (12), 2235-2238.

7. Fisher, R. A., Statistical methods for research workers. In Breakthroughs in statistics, Springer: 1992; pp 66-70. 